\title{
¿QUIÉN HIZO IMAGINAR A EINSTEIN DESTELLOS DE LUZ EN LAS VENTANAS DE LOS TRENES VISTOS DESDE LOS ANDENES?
}

\author{
WHO MADE EINSTEIN IMAGINE LIGHT SPARKLES IN THE \\ WINDOWS OF THE TRAINS SEEN FROM THE PLATFORMS?
}

\author{
Marc Boqué ${ }^{1}$ \\ Universitat Oberta de Catalunya (España)
}

Recibido: 17-12-2014

Aceptado: 15-3-2015

Aı $\omega \nu \pi \alpha \iota \sigma \varsigma \varepsilon \sigma \tau \iota \pi \alpha \iota \xi \omega \nu, \pi \varepsilon \tau \tau \varepsilon \cup \omega \nu \pi \alpha เ \delta \circ \varsigma \eta \beta \alpha \sigma \iota \lambda \eta \iota$

La vida es un niño que juega, que mueve las piezas sobre un tablero: reino de un niño.

Heráclito, B 52 DK

\begin{abstract}
Resumen: A cierta distancia de las discusiones en las que está sumergida la física experimental a finales de siglo XIX, la figura de Albert Einstein emerge con fuerza en 1905 a raíz de la presentación, en su articulo Zur Eletrodynamik Bewegter Körper, de un problema que hasta la fecha había hecho incompatibles el principio de relatividad galileo-newtoniano y la de ley de propagación de la luz. En la primera parte de nuestro artículo, tratamos de abrir el dilema presentado por el joven físico a partir de un ejemplo que recoge esta paradoja y mediante su desarrollo, intentamos encontrar en la segunda «las condiciones de posibilidad» que permitieron, no solamente desarrollar una teoría de la relatividad especial que cambiaria los viejos conceptos de espacio y tiempo de la física clásica y moderna, sino también una nueva forma de pensar que influenciada por el empirismo de la sospecha de Mach y la facultad de la imaginación desarrollada por Hume, abriría un nueva manera de mirar la realidad para el pensamiento contemporáneo.
\end{abstract}

Palabras clave: Einstein-Relatividad- Espacio- Tiempo.

[1] (mboquep@uoc.edu) Licenciado en Filosofia por la Universidad de Barcelona y doctorando por la Universidad de Zaragoza, realizando una tesis sobre el problema de la expresión en Giorgio Co1li. Ha cursado estudios de Física por la Universidad Nacional de Educación a Distancia (UNED) y actualmente es profesor colaborador en la Universitad Oberta de Catalunya (UOC), donde imparte dentro del Grado de Humanidades y el de Antropologia y evolucion humana, las asignaturas del área de Pensamiento filosófico y científico moderno y contemporaneo. 


\begin{abstract}
Located at a distance of discussions that is immersed the experimental physics in the late nineteenth century's the figure of Albert Einstein emerges strongly in 1905 following the publication in his article Zur Eletrodynamik Bewegter Körper of un issue in the form of paradox that had made incompatible Galilean-Newtonian's principle of relativity and propagation of light law to date. In the first part of our paper we try to open the question presented by the young physicist on the basis of an example and through their development to found in the second part "the conditions of possibility" that make it possible not only the development the special theory of relativity that would change the old concepts of space and time, but also a way of thinking which influenced by suspicion's empiricism of Mach and the power of imagination developed by Hume, it would open up a new way of looking the reality for contemporary thinking.
\end{abstract}

Keywords: Einstein- Relativity- Space- Time.

\title{
1. Imaginar un problema
}

Es seguro que también tú, te has dejado llevar alguna vez por los derroteros por los que nos invita a circular esta facultad nuestra que es la imaginación. Permíteme así, y siguiendo las reglas de su juego, que te coloque ahora con este ejemplo dentro de un vagón de tren que se desplaza a cierta velocidad constante sobre la vía por la que circula. Y no queriéndote privar del derecho a una imagen particular -quizás ésta ya demasiadas veces explotada en tus clases de física escolar-, amenizarla concibiéndote como un viajero poseedor de un billete, que en este momento en que cae la noche, te da derecho a estar cómodamente tumbado sobre una cama típica de compartimento, en un tren cuyo techo, eso sí, y como curioso deseo del decorador, ha estado forrado con un espejo en el que en esta posición te ves reflejado.

La noche es larga y tienes una pequeña linterna por si apremia alguna necesidad... Imagina que ahora la enciendes y proyectas su luz sobre el techo, y que atrapado por este tipo de pensamientos que aparecen en estas horas en que caemos en el sueño, piensas en su recorrido. Te dices que, por alguna razón, este centelleo viaja desde tu pequeño proyector hacia arriba, para reflejarse en el espejo y luego volver al punto del que partió, y esto te lleva, ya seducido en el pensamiento que abraza tu propuesta, a

Thémata. Revista de Filosofía Nº5 (2016) pp.: 63-86. 
pensar en el tiempo que este destello ha tomado para recorrer semejante distancia. Podrías dibujar la situación, si te apeteciera, de esta manera:

(fig. 1)

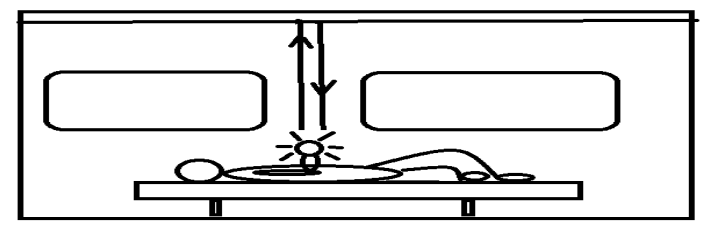

Supón ahora que yo, que te he puesto en esta situación, miro el vagón que ahora pasa contigo dentro- y todavía con tus cavilaciones-, desde un largo andén exterior, viendo un destello a través de los cristales producto de tu juego de proyección con la linterna, subiendo hasta la altura del techo, pero esta vez dibujando ante mi puesto de referencia una trayectoria que solo puedo dibujar para que puedas ponerte en mi situación:

(fig. 2)

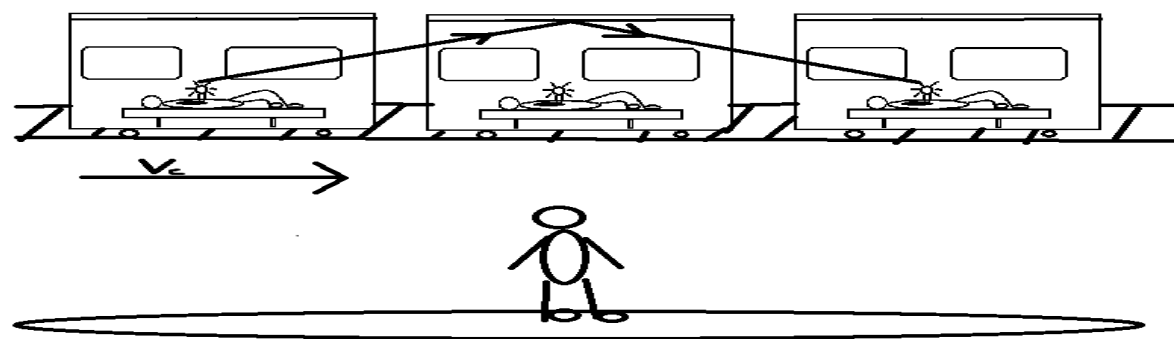

$x_{1} \quad x_{2}$

Como ves, nuestros dibujos no coinciden a la hora de precisar la trayectoria que recorre el mismo rayo de luz, aún más, el retorno de la señal, que en tu caso vuelve al mismo punto de partida, no lo hace desde mi apeadero; yo no veo en definitiva el mismo recorrido que tú. Es mi experiencia distinta de la tuya, ¿pero alguna de las dos equivocadas? Y no obstante, no parece que podamos dar preferencia a ninguna de nuestras posiciones si queremos respetar este principio que nos predestina a la relatividad de nuestras observaciones; principio, que ahora también intuirás 
problemático, pues, ¿no tendría que ser compatible esta perspectiva ya convertida en ley con cualquier otra, como por ejemplo la que nos dice que la luz debería propagarse siempre de la misma manera independientemente de nuestro lugar de referencia? Sin embargo, son ambas premisas, por ser una principio y otra ley, las que tanto tú como yo deseamos que gobiernen la naturaleza.

\section{El joven Einstein: la paradoja fuera de la academia}

Aunque también surgido de nuestra fabulación, bien podría ser el anterior fragmento un ejemplo del problema al que se enfrentaba el joven Einstein en sus horas libres como encargado de la oficina de patentes de Zúrich. En los años anteriores a 1905, fecha de la publicación de los artículos que cambiarían la historia de la física ${ }^{2}$, es aún un total desconocido en el mundo académico -se ha licenciado en el Instituto Politécnico de Zurich, pero no ha encontrado trabajo en ninguna universidad-, y por ello, disfruta aún del reto de enfrentarse a los problemas que su mente libremente le propone. Con todo, sus «juegos» necesitan de unas reglas y éstas, a falta de unos laboratorios donde ponerlas en práctica, las encuentra cerca del terreno solitario de la lógica. No es que durante su juventud desconozca ciertos experimentos ópticos y electromagnéticos que recorren su época, -aunque su interés o información pueda ser considerado como reducido ${ }^{3}$-, solo que los problemas a los que se quiere enfrentar, no se infieren de éstos, pues aspiran a partir de postulados.

Dos premisas recorren entonces sus cavilaciones; en primer lugar, quiere otorgar a la primera ley de Newton la categoría de ley aplicable a cualquier campo de la física, cosa que vista desde el ejemplo de nuestro viajero de la linterna, significaría que ninguno de los dos observadores del destello estaría justificado para afirmar su propio movimiento o reposo. ¿Alguien le puede asegurar al viajero del compartimento, cuando a medianoche se despierte alertado por el silbido de un tren que se cruza, que no duerme en la habitación de su casa? ¿Cómo distinguir el movimiento del

[2] Nos referimos a los artículos publicados en 1905 en Annalen der Physik: Über einen die Erzeugung und Verwandlung des Lichts betreffenden heuristischen Gesichtspunkt. Annalen...17, 1905 pp. 132-148, Über die von der molekularkinetischen Theori der Wärme geforderte Bewegung von in ruhenden Flüssigkeiten suspendierten Teilchen . Annalen...17, 1905 pp. 549-550, Zur Eletrodynamik Bewegter Körper. Annalen 17, 1905 pp.891-921, Ist der Trägheit eines Körpers von seinem Energieinhalt abhängig? Annalen... 18,1905 pp. 639-641, Über die von der molekularkinetischen Theori der Wärme geforderte Bewegung von in ruhenden Flüssigkeiten suspendierten Teilchen. Annalen...19,1906 pp 371-381.

[3] Ver Sánchez Ron, JM. El origen y desarrollo de la relatividad. Madrid: Ed. Alianza, 1983 p. 61 .

Thémata. Revista de Filosofía ํ53 (2016) pp.: 63-86. 
reposo si este no implica una modificación de la velocidad donde intervenga la fuerza que nos hace conscientes de donde estamos? ${ }^{4}$

Einstein se empeña en respetar para empezar «una ley fundamental de la mecánica galileo-newtoniana conocida como ley de la inercia que afirma, como sabemos, que un cuerpo alejado de otros persiste en su estado de reposo o movimiento rectilíneo uniforme ${ }^{5}$ y para bien o para mal, es esta falta de distinción entre dos estados equivalentes, resumida por la primera ley newtoniana ${ }^{6}$, la que lo destina a reconocer cierta relatividad al que él le otorga el nombre de restringida. Reconozcámoslo, es imposible diseñar experimento alguno para diferenciar estos dos estados; las leyes que experimentemos serán las mismas, al menos, para los sistemas que se mueven unos respecto a otros inercialmente; iguales en consecuencia tanto si nos movemos tumbados dentro de la cama del vagón desplazado en velocidad rectilínea constante, como si restamos de pie sobre el terraplén, y por tanto lo que veamos, por muy distinto que sea, tendrá la misma legitimidad: dejad caer la linterna que sostenéis tumbados sobre la cama y veréis como ésta cae al suelo del furgón en línea recta debido a la gravedad, podría decir Einstein, mirad esta acción ocurrida en el tren en movimiento desde la andana y veréis que también, y como consecuencia de la misma ley gravitatoria, describe ahora ante vosotros una trayectoria parabólica.

Nada podemos hacer, en consecuencia, para determinar si el tren se está moviendo y la vía está en reposo, o es al revés, y esta relatividad parece condenar a la imposibilidad la mirada privilegiada. Las leyes de la física, tienen que ser para el físico lógico con su principio, validadas tanto para un sistema de coordenadas asociado al andén, como para otro asociado al vagón donde viaja el personaje de la linterna. Y aquí reside la terquedad de su primera premisa.

Muy seriamente se toma el joven Einstein este postulado relativista que, sintéticamente, implica el deseo de encontrar la universalidad de unas leyes que sirvan para cualquier sistema inercial, y que aún así, nos condena a la relatividad de nuestra experiencia observacional... Aunque también, ya asumida esta primera regla para su juego, intuya por entonces, la problemática que a este requisito presenta la que quiere tomar como segunda; una ley desarrollada por la física moderna y que todo

[4] La segunda ley de Newton dice: «El cambio de movimiento es proporcional a la fuerza motriz impresa y se hace según la línea recta en que se imprime la fuerza».» Newton, I. Principios matemáticos de la filosofia natural. Madrid: Editora Nacional, 1982 p. 237.

[5] Einstein, A. La teoría de la relativitat $i$ altres textos. Barcelona: Editorial Pòrtic/Eumo editorial, 1998 p. 12.

[6] La primera ley de Newton dice: «Todos los cuerpos perseveran en su estado de reposo o de movimiento en línea recta, salvo que se vean forzados a cambiar este estado por fuerzas impresas». Newton, I. Principios cit., p. 237.

Thémata. Revista de Filosofía №53 (2016) pp.: 63-86. 
estudiante, sabe o cree saber, y que consiste en que esta propagación tiene lugar en línea recta y a una velocidad constante ${ }^{7}$, nos justificará.

Efectivamente, se desprende de la ley de propagación de la luz que nuestro destello tiene adscrita una velocidad de $3 \times \mathrm{m} / \mathrm{s}$. Ahora bien, si es así, ¿no se tendría que referir este proceso de propagación, como cualquier otro, a un sistema desde el cual mirarlo ${ }^{8}{ }^{8}$ como consecuencia, ¿no tendrían que calcular distintas velocidades para el destello de luz nuestro espectador tumbado en la cama interior del vagón y el que mira el paso de éste a cierta velocidad desde el andén- como hasta ahora pensábamos-, según el teorema de adicción de velocidades?'. Y sin embargo y con ello, la ley de propagación que precisamente como tal, otorga una velocidad constante a la luz, ya no sería posible en cualquier sistema de referencia inercial: desde el andén mediríamos una velocidad del destello de luz distinta que desde el interior del vagón, cosa que chocaría de frente con el principio de relatividad antes aceptado, el cual exige precisamente, que cualquier ley sea válida desde cualquier cuerpo de referencia inercial que se quiera tomar.

En efecto, las revolucionarias investigaciones teóricas de H. A. Lorenz sobre los procesos ópticos y electrodinámicos en cuerpos en movimiento, habían demostrado que las experiencias en este dominio conducían necesariamente a una teoría de los

[7] «No hay ninguna ley más simple que la propagación de la luz en el vacío. Todo estudiante sabe, o cree saber, que esta propagación tiene lugar a una velocidad de $C=300.000 \mathrm{~km} / \mathrm{s}$. Einstein, A, La relatividad cit., p. 15.

[8] Hasta la fecha, la teoría ondulatoria de la luz, solo aceptaba este principio de propagación de la luz para un sistema de referencia en reposo que contuviera el éter en el cual se propagaran las ondas electromagnéticas. Al respecto, ver nuestro artículo, punto 3.

[9] El teorema de adicción de velocidades nos propone la medición de la velocidad respecto al sistema de coordenadas desde la que la realicemos. Así si por ejemplo, dentro de nuestro vagón el viajero tumbado en la cama se levanta y empieza a andar a lo largo del interior del vagón éste podrá decir que se desplaza a un velocidad $\omega$. ¿Pero a qué velocidad dirá que se está moviendo este pasajero un espectador que lo ve pasar desde el andén viajando en este vagón que se desplaza también a una velocidad v? Sin duda diríamos que para éste observador atento al paso del tren a:

$$
\mathrm{V}=\mathrm{v}+\omega
$$

¿No es cierto? Y sin embargo si ahora el que se mueve a lo largo del vagón es nuestro rayo de luz no podemos aplicar la adicción anterior, en este caso

$$
\mathrm{V}=\mathrm{c}+\omega
$$

Siendo C, la velocidad de la luz, $3 \times 10^{8} \mathrm{~m} / \mathrm{s}$

a menos que dejemos de respectar una ley que nos dice que la velocidad de la luz es una absoluto independientemente del sistema de referencia desde el cual se lo quiera medir.

Thémata. Revista de Filosofía $\mathrm{N}^{\circ} 53$ (2016) pp.: 63-86. 
procesos electromagnéticos que tenía como consecuencia irrecusable la ley de constancia de la velocidad de la luz en el vacío. Pero también por ello, los principales teóricos estaban dispuestos a abandonar el principio de relatividad, aunque no se hubiese encontrado ni un solo experimento que lo contradijese. ${ }^{10}$

Para el joven Einstein, entonces, superar la paradoja consistirá en hacer compatible la tensión entre estos dos requisitos; el principio de relatividad y la constancia de velocidad de la luz y, ¿no resultará esto un reto apasionante para el joven que entonces estaba fuera de la física académica?

\section{Resolver la incompatibilidad a través del cuestionamiento de un concepto avalado por el sentido común}

En este problema autoimpuesto andaba Einstein en los descansos de su trabajo en la oficina de patentes y también fuera le seguía persiguiendo. Pero la lucha con los retos a los que voluntariamente nos enfrentamos toma a veces desenlaces repentinos, y en el caso de Einstein se dio, como el mismo nos cuenta en una conferencia de 1922, gracias a la visita periódica que por aquellos años de juventud realizaba a su buen amigo Michele Besso ${ }^{11}$. Aquejado por sus dilemas sobre rayos y trenes, se presentó a uno de estos encuentros confesando abiertamente a su amigo el dilema que le azotaba, y aún bajo el marco de la puerta desde el cual le recibió, le confesó a éste que esa vez "había traído un problema con él». La enigmática presentación debería crear cierta expectativa en el interlocutor y unos asociados segundos de silencio, tiempo que según nos cuenta el propio Einstein él mismo lleno para reflexionar sobre las propias palabras que acababa de decir; las mismas que tendrían que iluminar, repentinamente, su ansiado desenlace.

Mi solución en realidad se refería al concepto de tiempo. Es decir, el tiempo no puede ser absolutamente definible por sí mismo... ${ }^{12}$

¿No sería una condición ineludible para cualquier concepto el tener que ser defi-

[10] Einstein, A. La relatividad cit., p. 60.

[11] Conferencia en Kyoto el 14 de diciembre de 1922. La historia es extraída de las notas tomadas por Jun Ishiwara. Recogidas por Norton, John D. (2010) How Hume and Mach Helped Einstein Find Special Relativity. In: Discourse on a New Method: Reinvigorating the Marriage of History and Philosophy of Science. Open Court, Chicago and La Salle, IL, p. 359-386. ISBN 9780812696622. Recuperado el 20 de julio de 2014, de: http://www.pitt.edu/ jdnorton/ papers/HumeMach.pdf

[12] Ibídem, p. 11.

Thémata. Revista de Filosofía №53 (2016) pp.: 63-86. 
nido en conexión con los principios que configuraban el problema que había traído con él? Es decir, lo que seguramente intuyo Einstein en las propias palabras proferidas a su amigo es que un concepto como el de tiempo quizás no era tan distinto a un problema que se pudiese llevar de un lugar a otro, y que por tanto, tampoco éste podía ser independiente de una experiencia en particular, ni definido sin establecer una implicación con otros conceptos ya probados en tal situación.

Einstein, después de la visita a su amigo había decidido sospechar del concepto habitual que tenemos del tiempo, un tiempo absoluto que pasa a la vez para todos, pero concepción que nunca había sido probada empíricamente. Se necesitaba, por tanto, una verdadera redefinición de este concepto que solo el sentido común avalaba, y Einstein, la quiso encontrar de vuelta a su casa, precisamente vinculándola al concepto de simultaneidad, pues:

Hemos de tener en cuenta que todo juicio en que interviene el tiempo es siempre un juicio sobre sucesos simultáneos. ${ }^{13}$

Pero si este concepto de simultaneidad podía pasar ahora el filtro autoimpuesto, sin duda su camino transcurría por encontrar un método experimental para su medición, y aquí Einstein lo encontró apoyándose en ese postulado hasta ahora inviolable para la física y que justamente él quería utilizar como segunda premisa. Efectivamente, sólo a través de esta ley vinculada a la constancia de la velocidad de la luz podría sentenciar después que dos acontecimientos que ocurren en dos puntos A y B son simultáneos, y por tanto comparten el mismo tiempo, si y sólo sí, «el tiempo que necesita la luz para ir de A a B es igual al tiempo que necesita para ir de $\mathrm{B}$ a $\mathrm{A} »$.

Einstein en la puerta de la casa del amigo pareció entenderlo: es a costa del desvanecimiento de un supuesto y su obligada redefinición respecto a otros ya sólidamente probados que se pueden abrir nuevas preguntas, pero también resolver antiguos problemas arrastrados. Y es precisamente esta visión la que también ahora nos puede ayudar a resolver nuestro problema planteado al inicio de este artículo.

Bajo esta redefinición de tiempo, ¿será posible aún éste tiempo común para dos acontecimientos tomados desde dos experiencias distintas? O del modo particular de nuestro ejemplo imaginado: ¿podrán seguir compartiendo el mismo tiempo la experiencia de ver el rayo desde el interior del vagón y su visualización desde el andén?

Dejémonos llevar por el juego de Einstein, atrevámonos a poner en cuestión un concepto que nos dice que el tiempo ha de transcurrir de manera absoluta y adecuémoslo a la nueva definición que lo vincula el

[13] Einstein, A, La relatividad cit., p. 90.

Thémata. Revista de Filosofía №53 (2016) pp.: 63-86. 
postulado de la constancia de velocidad de la luz como condición para su medición, y haciendo de este principio una ley compatible en cualquier situación como nos dice el principio de relatividad, volvamos sobre nuestros destellos de luz:

¿Qué tiempo tarda en recorrer la distancia el rayo que va desde la linterna al techo y vuelve a la posición inicial?

Visto desde el interior del vagón nos atrevemos a decir ahora, y bajo la nueva definición, que el tiempo que tarda el destello de luz en recorrer la distancia en la fig. 1 es:

$$
\Delta \mathrm{t}^{\prime}=2 \mathrm{D} / \mathrm{c}
$$

Siendo D la distancia entre la posición de la linterna y el techo y C la velocidad de la luz.

A continuación miremos desde el andén con la libertad que nos permite la nueva perspectiva; el vagón pasa a una velocidad V por delante del espectador parado sobre él. Éste ve como el vagón se mueve junto con el espejo, y el rayo que sale de la linterna desde una posición $\left(x_{1}\right)$ se refleja en otra $\left(x_{2}\right)$. Los dos acontecimientos (salida del destello y llegada del destello) se producen a una distancia horizontal que no resulta difícil de determinar:

\section{$\mathrm{V} \Delta \mathrm{t}$}

Y podemos ver en la fig. 2 que el recorrido de la luz es más largo que en fig. 1, lo que no contradice el principio de relatividad. Sin embargo, respetemos el segundo postulado ahora, y si nuestro destello de luz se propaga a la misma velocidad independientemente que se mire desde el interior del tren o desde el terraplén- principio contenido en la nueva definición del tiempo- tendremos que, el intervalo de tiempo del rayo de luz experimentado desde la visión del terraplén, por fuerza deberá emplear más tiempo 
en recorrer una distancia mayor que el observado cómodamente tumbado en la cama del interior del vagón. Contrastemos las distancias:

(Fig. 3)

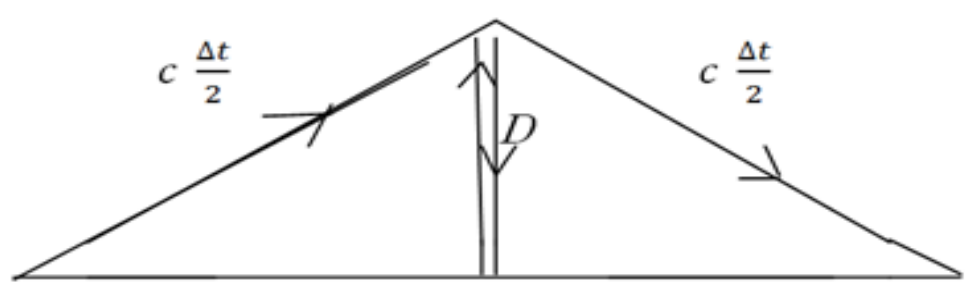
$v \frac{\Delta t}{2}$
$v \frac{\Delta t}{2}$

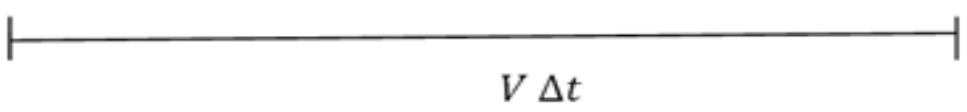

Siendo $C$ la velocidad de la luz

Y apliquemos sencillamente el teorema de Pitágoras:

$((\mathrm{c} \Delta \mathrm{t}) / 2)^{2}=\mathrm{D}^{2}+((\mathrm{v} \Delta \mathrm{t}) / 2)^{2}$

que resulta operando:

$\Delta \mathrm{t}=2 \mathrm{D} / \sqrt{ }\left(\mathrm{c}^{2}-\mathrm{v}^{2}=2 \mathrm{D} / \mathrm{c} \quad\left[1 / \sqrt{ }\left(1-\left(\mathrm{v}^{2} / \mathrm{c}^{2}\right)\right]\right.\right.$

Thémata. Revista de Filosofía №53 (2016) pp.: 63-86. 
Y substituyendo (1), $\Delta \mathrm{t}^{\prime}=2 \mathrm{D} / \mathrm{c}$, para obtener:

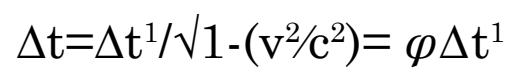

Donde partiendo de la las reglas del juego que Einstein nos ha querido proponer, y aplicadas a nuestro ejemplo, encontramos una diferencia $\varphi$ entre el tiempo que calcularía que tarda entre ir y volver el destello emitido por su linterna el pasajero tumbado sobre su cama en el interior del vagón de tren $\left(\Delta t^{1}\right)$, y el tiempo que calcularía el espectador del andén para el mismo acontecimiento $(\Delta \mathrm{t})$. Dos tiempos distintos para cada uno de los observadores; dos observaciones donde ahora podemos confirmar que ya funcionan las dos leyes de la naturaleza antes incompatibles. Y sin embargo, la paradoja entre el principio de relatividad y el de propagación de la luz en el vacío se ha resuelto lógicamente al precio de poner entre paréntesis un concepto, el del tiempo absoluto, que ya no puede ser común o simultáneo como se daba por sentado, y que ahora, tiene que pasar por el cedazo de la experiencia particular.

\section{Conocimiento y presunción de conceptos físicos ilusorios}

Nos podría objetar alguien, no sin fundamentada razón, que quizás hemos otorgado en nuestro inicio una posición demasiado exterior a un Einstein que no obstante resolvió la incompatibilidad planteada en nuestro ejemplo, y a través de su famoso artículo Sobre la electrodinámica de los cuerpos en movimiento ${ }^{14}$, con la ayuda de unas ecuaciones de transformación que ya había desarrollado Lorenz a partir de las contribuciones de Maxwell en el electromagnetismo y los resultados del experimento de Michelson Morley ${ }^{15}$. Al respecto, Einstein, llego a decir en una entrevista

[14] Ver nota 2.

[15] Fue en el año 1887, cuando el físico americano A. A. Michelson y su ayudante E.W. Morley realizaron el famoso experimento. Si Fizeau unos años antes había podido observar la influ-

Thémata. Revista de Filosofía №53 (2016) pp.: 63-86. 
mantenida en febrero de 1950 con R. S. Shankland, que cuando publicó en 1905 el artículo donde se anunciaba la relatividad, no conocía aún el experimento de Michelson-Morley más que a través de los escritos de Lorenz. Estas afirmaciones quedaron desmentidas posteriormente gracias a la aportación de J. Stachel, que en 1987, demostró a través de diversas evidencias, que en este caso la memoria se había mostrado poco condescendiente con un ya viejo Einstein ${ }^{16}$. Falta de memoria, pues, para las fechas y detalles que no se ajustaban a una realidad acontecida hacía demasiados años - el estudiante alemán efectivamente conocía de primera mano y como el resto de alumnos, el experimento de 1887-, aunque no creemos que esto influenciara en la esencia de la práctica solitaria de un joven que nunca utilizó las ecuaciones y experimentos del pasado con la misma intención que los habían recogido la teoría de sus antecesores ${ }^{17}$.

Es importante destacar que en la teoría de Maxwell-Lorenz (el segundo postulado de constancia de la luz que utiliza Einstein) sólo tenía carácter de axioma en sistemas de referencia en reposo con respecto al éter. En este punto se halla una de las mayores diferencias entre las teorías de Einstein y de Lorenz. Según la teoría de este último dos sistemas de referencia en reposo (uno en reposo en el sistema del éter y otro en movimiento inercial) están relacionados por sus transformaciones (las de Lorenz) que no forman grupo y no lo forman porque como el éter nunca se mueve, no tiene sentido la transformación inversa del sistema de referencia en movimiento al sistema en reposo del éter..$^{18}$

Tanto Maxwell como Lorenz pertenecen aún a una tradición que sostiene la existencia de un espacio absoluto de herencia newtoniana; con realidad ontológica, uniforme y substancial (actúa sobre los objetos sin que éstos actúen sobre él). Cumple esta concepción del espacio con la ley de inercia, aunque a final de siglo XIX y principios del XX, también esté ocupado necesariamente por un éter capaz de transmitir las nuevas ondas lumínicas que recientemente han abierto la nueva explicación sobre la naturaleza de la luz. Construida entonces ésta a partir de una teoría de ondas, está sometida a una ley de constancia de velocidad, pero solo

encia de una corriente rápida de la luz que se propagaba a su través. ¿No se podría también observar el mismo efecto del movimiento de la Tierra en el espacio sobre la velocidad de la luz medida en su superficie?

[16] Sánchez Ron,J. M. El Origen cit., p. 62.

[17] Antes de que se publicara el articulo de Einstein Sobre la electrodinámica de los cuerpos en movimiento, Hendrick A. Lorenz y George F. Fitzerald habían intentado dar cuenta del resultado nulo del experimento de Michelson Morley suponiendo que las distancias en la dirección del movimiento se contraían de acuerdo a la ecuación $L=1 / \varphi \quad L_{p}=L_{p} \sqrt{ }\left(1-v^{2} / c^{2}\right.$, donde vemos que el factor $\varphi$ coincide con la diferencia de tiempos de nuestro ejemplo.

[18] Sánchez Ron,J. M. El origen cit., p. 67.

Thémata. Revista de Filosofía №53 (2016) pp.: 63-86. 
respecto a sistemas de referencia anclados en esta substancia etérea en reposo; sistemas que formarían, efectivamente, un grupo separado de los que no lo hacen.

La transformación de Lorenz podríamos decir que tiene una dirección, mientras que el desarrollo de Einstein en la teoría de la relatividad recordemos que parte de un postulado relativista ineludible, que establece que todos las transformaciones han de formar un mismo grupo que contenga a todos los observadores que se muevan unos respecto a los otros en movimiento constante. De hecho, es la experiencia la que no puede diferenciar entre sistemas inerciales, y así el axioma del segundo postulado, el de la constancia de la velocidad de la luz, ha de ser válido para todos ellos. En definitiva, si para la física experimental en la que se crio nuestro autor, el comportamiento ondulatorio de la luz y la ley de constancia de su velocidad intentaban justificar precisamente la existencia de un material que daba legitimidad a un espacio aún absoluto, para nuestro físico, cuya mirada creemos que observa bajo un nuevo modelo teórico, el éter es sencillamente innecesario para su problema. Él, toma de la teoría de Maxwell un principio, pero solo para radicalizarlo hasta convertirlo en postulado, aunque esto implique que:

Para que esto esté en armonía con la equivalencia de los sistemas inerciales (principio de la relatividad especial) deba abandonarse el carácter absoluto de la simultaneidad, además, las transformaciones de Lorentz para las coordenadas de tiempo y espacio valen para la transformación de un sistema inercial a otro. El contenido total de la teoría de la relatividad especial está incluido en el siguiente postulado: las leyes de la naturaleza son invariantes con respecto a las transformaciones de Lorentz. ${ }^{19}$

Es precisamente este cuestionamiento del carácter absoluto de un concepto- en este caso el de tiempo absoluto- puesto en entredicho por una ley de pretensión universal (las leyes de la naturaleza han de valer en cualquier situación) $)^{20}$, el que constituye la piedra de toque ante la física anterior. Hasta la fecha, el uso «del viejo» concepto de tiempo había requerido la presunción tácita de que los juicios de simultaneidad eran independientes del observador o marco de referencia; su propia percepción. Y sin embargo, la aportación de Einstein va ligada a este cuestionamiento. Claro

[19] Einstein, A. Sobre la teoría de la relatividad y otras aportaciones. José M. Álvarez y Ana Goldar (Trads.) Madrid: Editoral Sarpe, 1983. P. 181.

[20] Esta pretensión siguió tomando cuerpo hasta 1915, año en que Einstein fue capaz de formular satisfactoriamente las ecuaciones de de campo de la gravitación y proponiendo la teoría de la relatividad general, que extendía las leyes de la naturaleza a sistemas de referencia fuera cual fuera su estado de movimiento.

Thémata. Revista de Filosofía №53 (2016) pp.: 63-86. 
que el uso de un concepto en una teoría física- y seguimos con el de tiempo absoluto- requiere típicamente de algún tipo de presunción de hechos. El peligro sobre el que nos quiere alentar Einstein, en todo caso, surge en cuanto, precisamente, estos conceptos se desligan de ellos y nos comprometen a presunciones físicas falsas que nos introducirían sin saberlo, en una especie de conocimiento "ilusorio» a priori; éste entra en nuestra teorización a través de una elección de conceptos y no a través de la investigación empírica de la presunción ${ }^{21}$. Esta sospecha, es vital para el pensamiento de un autor que, desde niño, a perseguidos rayos de luz y queriendo así abrir problemáticas, se ha alejado voluntaria e involuntariamente de los círculos oficiales.

\section{Mach: poner los conceptos cerca de la tierra o purgar}

Fundamentada la aportación de Einstein en una cierta toma de distancia, nos toca ahora indagar en esta mente interrogadora cuya singular aportación también creemos que se funda en unas condiciones de posibilidad. A tales efectos, su propia autobiografía podría ser un buen memorando, que sin embargo consideramos demasiado alejada de los acontecimientos que marcaron el nacimiento de la teoría de la relatividad. Así, buscando testimonio un poco más cercano a su momento fundacional, encontramos la carta que Einstein dirigió a Schlick -entonces Privatdozent de Filosofía en la Universidad de Rostock- desde Berlín, el 14 de diciembre de 1915 y donde apuntando a los comentarios filosóficos de su interlocutor le comentaba:

Su presentación de que la teoría de la relatividad se sugiere en el positivismo, aunque sin que la requiera [necesariamente], es... muy correcta. En esto también vio usted correctamente que esta línea de pensamiento tuvo una gran influencia en mis esfuerzos, y más concretamente, E. Mach, e incluso más Hume, cuyo Tratado sobre la naturaleza humana había estudiado con avidez y con admiración poco antes de descubrir la teoría de la relatividad. ${ }^{22}$

[21] Norton, John D. How Hume and Mach Helped Einstein Find Special Relativity. In: Discourse on a New Method: Reinvigorating the Marriage of History and Philosophy of Science. Open Court, Chicago and La Salle, IL, 2010 pp. 359-386. Recuperado el 29 de julio de 2014, de:http://www.pitt.edu/ jdnorton/papers/HumeMach.pdf

[22] Reproducida en Robert Schulmann,A. J. Kox, Michel Janssen y József Illy, eds., The Collected Papers of Albert Einstein, vol. 8 (The Berlin Years: Correspondence,1914-1917), Parte A (Princeton University Press, Princeton 1998), p. 220. El artículo de Schlick al que se refería Einstein es: Moritz Schlick, «Die philosophische Bedeutung des Relativitätsprinzips», Zeitschrift für Philosophie und philosophische Kritik 159, 1915 pp. 129-175

Thémata. Revista de Filosofía $\mathrm{N}^{\circ} 53$ (2016) pp.: 63-86. 
De la influencia de E. Mach mucho se ha comentado, sobre todo por parte de algunos destacando la aportación que su crítica a la concepción del espacio newtoniano tuvo directamente sobre Einstein y de otros, más osados, subrayando como sus propuestas personales ya rozaron incluso la teoría de la relatividad ${ }^{23}$. Por nuestra parte, sólo queremos centrarnos ahora en un punto muy concreto de su lectura epistemológica, que fue precisamente la que libremente decidió destacar Einstein en el obituario de muerte del autor austriaco ${ }^{24}$. Leemos en él:

Según Mach, la ciencia no es más que la actividad de comparar y de ordenar, según ciertos puntos de vista y siguiendo determinados métodos presentes en nuestra conciencia (...) Esta demostración del orden se acaba en la definición de conceptos abstractos y en el enunciado de las leyes que rigen su relación. Estos conceptos y estas leyes son elegidas de tal manera que juntas formen un sistema capaz de estructurar firmemente y claramente los datos que hace falta explicar. Se sigue que los conceptos solo tienen sentido en la medida en que alguien puede pueden designar claramente las cosas a las cuales se refieren y los puntos de vista en función del los cuales se refieren (análisis de conceptos). ${ }^{25}$

\section{Pero aún más:}

A menudo los conceptos que se han revelado útiles para ordenar la realidad adquieren tanta autoridad que olvidamos su origen terrenal y los consideramos realidades inmutables. Les damos entonces el nombre de «necesidades lógicas», «datos a priori», etc. Estos errores suelen impedir el progreso científico durante mucho tiempo. No tendríamos que considerar, entonces, un pasatiempo de desbagados el ejercicio de analizar conceptos consolidados por la tradición, y tendríamos que hacer por manera

[23] El mismo Einstein hace referència a la influencia de Mach en su autobiografia. Einstein, A. Autobiografía y escritos científicos. Barcelona: Biblioteca Universal del Círculo de Lectores, 1995 pp. 50-57.

[24] «¿Pero por qué un buen investigador de la naturaleza se ha de ocupar de la teoría del conocimiento? ¿No sería mejor que trabajara en otra especialidad? Es la opinión que oigo de algunos de mis colegas o el sentimiento que me parece descubrir en muchos de ellos. No comparto en nada esta opinión. Cuando pienso en los estudiantes que he conocido en mis clases, me doy cuenta que los mejores, los que se distinguen por su independencia de juicio y no solamente por su saber, manifestaban un vivo interés por todo lo que hacía referencia a la teoría del conocimiento. Tomaban parte en discusiones sobre los fines y los métodos de las ciencias, i la determinación con que defendían su punto de vista demostraba claramente la importancia que atribuían a esta cuestión. ¿Pero, puede ser que a alguien le extrañe?» «Ernst March», Physikalische Zeitschrift, 17 (1916) 101-104; el texto ha estado reproducido dentro de The collected Papers of Albert Einstein, vol.6: The Berlin Years (1914-1917), Princeton University Press, Princeton, 1996, pp. 277-282.

[25] Ibídem, p. 227.

Thémata. Revista de Filosofía Nº5 (2016) pp.: 63-86. 
de descubrir las circunstancias en que se originaron y cuáles son los hechos de experiencia a los que se refieren. De esta manera, haríamos que perdiesen la autoridad excesiva que habían adquirido. Alguien los ha de eliminar cuando su existencia es injustificable, esmerar cuando su relación con los datos es defectuosa, reemplazarlos por otros cuando, por el motivo que sea, optamos por un nuevo sistema. ${ }^{26}$

Más allá de su positivismo, queda limpio en estas notas de Mach, que hay un denominador común para la idea que éste se hace de la ciencia, y es que en el fondo, no se rige ésta más que mediante la comparación y arreglo ordenado de los datos contenidos en nuestra conciencia; información que en todo caso, da paso a unos conceptos que en su uso habitual, y a menudo, olvidamos que necesariamente partieron precisamente de hechos de experiencia. El gran merito hallado por Einstein en su colega parece relacionado con el reconocimiento de que Mach hubiera sido capaz de ver que los grandes problemas físicos, antes que ser primordialmente de naturaleza deductivo-matemática, eran anteriormente fundamentados sobre principios básicos que la tradición desde hacia tiempo no había puesto en duda ${ }^{27}$. La influencia de un autor sobre el otro parte pues de la necesidad de poner "en tierra» estos conceptos, de analizarlos hasta poder justificarlos empíricamente, o si no se llegara al caso, a eliminarlos para substituirlos por otros que sí cumplieran estas condiciones.

Esta "purgación» del concepto de tiempo es sin duda un punto importante de partida para el autor de la relatividad especial; la puesta en cuestionamiento de los a priori con los que tanto combatió Einstein mucho le debe a $\mathrm{Mach}^{28}$. Aunque sospechamos, no obstante, que esta aportación sólo sea suficiente para dar cuenta de un método que sirva para legitimar el descubrimiento científico, mientras que nuestro ejemplo, necesite algo más para resolverse.

[26] Íbídem, p. 227.

[27] Para Mach las matemáticas tienen un carácter inminentemente práctico que solo toma relevancia respecto a la ciencia en general cuando nuestro pensamiento consigue reproducir los hechos sensibles que estas describen. Mach, Ernst. Análisis de las sensaciones, Barcelona, Alta Fulla, 1987 pp. 277-278.

[28] El autor representante por excelencia de este pensamiento de los a priori es Kant para Einstein. Ver a al respecto Einstein, A. «Kant» en Física i realitat (i altre escrits filosòfics). Barcelona: Obrador Edéndum S.L, 2005. 


\section{La insuficiencia de la sospecha para fundamentar la ciencia}

Creía Einstein que los físicos que lo habían precedido, al menos durante los siglos XVIII y XIX, no habían utilizado para fundamentar sus teorías físicas concepto alguno que no pretendieran descubrir en la naturaleza. En 1933, y ya con el bagaje de una teoría de la relatividad puesta en funcionamiento, afirmaba por ejemplo, que si bien Newton había sido el primer creador de un sistema global para la física, el inglés aun creía que los conceptos como el de masa, inercia y fuerza, que se insertaban en sus leyes fundamentales expuestas en los Principia, debían ser fundamentados en su sistema como resultados directos de la experiencia ${ }^{29}$. Es esta concepción, la que según Einstein, llevaba Newton a tener problemas con conceptos como los de espacio o reposo absoluto, que evidentemente, no se correspondían con nada de lo que pudiéramos tener una percepción mediante la experiencia.

Los científicos de aquella época estaban dominados por la creencia que los objetos y leyes fundamentales de la física no eran libres invenciones lógicas del espíritu humano, sino que a partir de experimentos podían ser obtenidas de la «abstracción» por una vía lógica. ${ }^{30}$

¿Pero es un concepto científico algo que puede ser extraído unívocamente de la experiencia? O dicho de otro modo, ¿podemos rendir cuentas de un conjunto de hechos percibidos a partir de un solo fundamento?

Einstein en 1933 ya ha sido reconocido en el mundo de la física por su desarrollo de la teoría que ha puesto contra las cuerdas algunos de los principios básicos de la física newtoniana, aunque esto es importante, no todos. Y por entonces, puede ya estar seguro de que dos explicaciones teóricas esencialmente distintas pueden concordar con una experiencia que siga siendo el inicio y el fin de nuestro conocimiento. Su propuesta para una metodología de la física consta de un doble trabajo:

Un sistema completo de física teórica consta de conceptos, de leyes fundamentales aplicables a estos conceptos y de consecuencias deducibles lógicamente. Estas deducciones se han de corresponder con cada una de nuestras experiencias (...) Por

[29] Texto de la «Hervert Spencer Lecture», pronunciada en Oxfort,, el 10 de junio de 1033, y publicada con el título: On the Method of Theoretical Physics, Clarendon, Oxford, 1933; el original alemán «Zur Methode der thoretischen Physik» se encuentra en http://www.alberteinstein.info. Einstein Archives Online, doc. 1-114

[30] Einstein, A. «El método de la física teórica» en Física i realitat (i altre escrits filosòfics). Barcelona: Obrador Edéndum S.L, 2005 p. 84.

Thémata. Revista de Filosofía №53 (2016) pp.: 63-86. 
otro lado, los conceptos y leyes fundamentales son invenciones libres del espíritu humano que no pueden encontrar justificación a priori ni en la naturaleza del espíritu humano ni en ningún lugar. ${ }^{31}$

Por una parte, encontramos en su propuesta epistemológica una tarea de contraste con la experiencia de corte muy empirista, e insubstituible para encontrar unos conceptos que debiéndose tomar como fundamento han de caer, sin duda, sobre la sospecha del atento investigador científico. Es la parte en que podríamos destacar las virtudes del descubridor. Pero por otra parte, se nos insiste en un carácter de invención libre que implica la posibilidad de acceder a nuestras percepciones únicamente a través de la limitación de la imagen que de ellas tomamos: conceptos y leyes, entonces, que aplicadas como obras humanas, nos permitirán obtener después una imagen completa del mundo por pura deducción lógico-matemática. Hablamos de un lado creativo que, aunque nombrado por Einstein de distinta manera durante su vida ${ }^{32}$, constituirá parte de esa capacidad que Mach no le supo aportar.

\section{Suponer es pensar}

Es dirigiendo la mirada a Hume que encontramos, por primera vez, una clasificación de los contenidos de nuestra mente que se mantiene inalterable durante toda la lectura de Treatise of Human Nature. Según el autor inglés- fervientemente leído por Einstein y sus amigos durante su época de estudiantes-, nuestro entendimiento contiene percepciones que se pueden reducir a dos tipos: impresiones o ideas ${ }^{33}$, y la diferencia entre ambas «consiste en los grados de fuerza y vivacidad con que inciden sobre la mente y se abren en nuestro pensamiento o conciencia» ${ }^{34}$. Nos cuenta a continuación, que son las primeras, las impresiones, esas sensaciones, pasiones y emociones que hacen aparición en nuestra alma de forma directa,

[31] Ídem, p. 84.

[32] Y creemos que es precisamente este "tomar como fundamento», en el sentido de elegir (Ibídem, "La recerca científica», pp. 65-67) el que en 1933 se convertirá en el concepto «libre invención». (Ibídem, «El mètode de la física». Pp. 73-74) y aun en 1941 la llegaremos a comprender en esencia como como la «obra humana» (Ibídem, "Fonamentació de la física». pp. 73-83).

[33] Hume, D. Tratado sobre la naturaleza humana. Madrid: Editora Nacional, 1981 p. 97.

[34] Ibídem, p. 87.

Thémata. Revista de Filosofía №53 (2016) pp.: 63-86. 
dejando en ella una impronta profunda, de mayor grado que las ideas, que solo son capaces de formar imágenes débiles de estas impresiones ${ }^{35}$.

Sintamos ahora, según esta interpretación, la luz que sale de la linterna en nuestro ejemplo y como ésta nos afecta los ojos; una luz que primero nos deslumbra y nos hace apartar la mirada; impresión que nos llega externamente y agranda la pupila y que es la percepción más simple por la que podemos ser afectados Sólo después de esta impresión esencial, podremos según Hume, retener esta imagen en nuestra mente, pensarla y convertirla en un nombre, por ejemplo ese «destello» que ilumina cada objeto en particular, o en una idea más compleja, si se desea, elaborada bajo el nombre de «onda», y que como condición, deberá conservar la misma velocidad tanto para el viajero tumbado en la cama como para el espectador que lo mira desde la andana, según la teoría de la relatividad... Reflejándose y refractándose, eso sí, para los dos observadores, construida, aún mas, por longitudes de onda que corresponden a los distintos colores, pero que de cualquier manera, solo la impresión causada en las pupilas sensoriales pude captar en una gradación del violeta al rojo.

De la impresión, simple o compleja, a la idea también, simple o compleja, el empirismo de Hume en el Treatise le debería parecer a Einstein sintetizado a partir del reto de hacernos imposible encontrar una sola imagen o concepto que no parta de la suma de sus fragmentos percibidos. Si miramos de esta manera al espació y el tiempo ¿qué podrían ser éstos en este contexto, sino ideas más o menos complejas, pero elaboradas a partir de una serie de impresiones?

Serie, que en el caso de la idea que tenemos del tiempo, solo puede haber estado construida, como cualquier otra, por sucesiones de impresiones o objetos mutables, porqué...

De igual modo que de la disposición de los objetos visibles y tangibles recibimos la idea de espacio, formamos la del tiempo en base a la sucesión de ideas e impresiones; el tiempo, por sí solo, no puede manifestarse ante la mente ni ser concebido por ella. ${ }^{36}$

Sí, sólo a partir de esta infranqueable clasificación que constituye una concepción epistemológica, nos podrá preguntar sagazmente el empirista: ¿puede ser causada la idea de tiempo por un objeto invariable? Igual que una línea ha de ser concebida a partir de las impresiones que nos causan los puntos que la forman, ¿se puede concebir un periodo de tiempo sin la sucesión de sus partes móviles? Película sin movimiento, como una

[35] Ibídem, p. 87.

[36] Ídem, p. 128.

Thémata. Revista de Filosofía №53 (2016) pp.: 63-86. 
colección de fotogramas parados, ¿existe el tiempo a partir de una imagen congelada?

Y a nosotros, irremediablemente, la pregunta que nos plantea Hume nos hace pensar otra vez en el rayo en que aun debe pensar el joven Einstein mientras lee en compañía el Treatise, o en un éter invariable como la imagen de la corte dormida que detiene el tiempo en el cuento de la bella durmiente; y estas imágenes imposibles para la percepción, también cuestionan la concepción apriorística de un tiempo que transcurra independiente del orden de una sucesión de instantes.

Existe, por ello, una manera de pensar en esta lectura de juventud, que invita al lector a abrir el campo de la potencialidad, fuerza o viveza, que parte de los datos de una impresión de la que no podemos inferir sus causas, y que sin embargo, posibilita el pensamiento. Éste solo puede surgir como capacidad para retener este dato que se nos escaparía en un mar de percepciones, sino fuésemos capaces de crear su impronta. Es por ello que Hume nos otorga, primero, la función de la memoria como condición para el desarrollo de nuestro entendimiento; instaurando ésta la condición de posibilidad para el mayor grado de vivacidad en la percepción: recuerda la impresión. Pero también la misma pone en orden y posición la imagen retenida, y por ello, puede revisar y fundamentar a partir de su análisis todos los pasos de los que nos hablaba a la vez Mach. Y en segundo lugar, una segunda facultad, la imaginación, que es la que nos permite un juego de facto, trastocando y alterando ese orden impuesto por la memoria a las ideas, combinándolas con sus operaciones de semejanza, contigüidad y causalidad $^{37}$ para: «formar monstruos y unir figuras y apariencias incongruentes, como le es concebir a los objetos más familiares y naturales.» ${ }^{38}$

\section{Y que sólo por ella:}

... mientras que el cuerpo debe arrastrarse laboriosamente sobre la superficie de un solo planeta, el pensamiento nos puede transportar instantáneamente a las regiones más lejanas del universo -e incluso más allá. Lo que nunca ha sido visto u oído puede no obstante ser concebido; nada está más allá del poder del pensamiento salvo lo que implica una absoluta contradicción. ${ }^{39}$

Corresponde, según Hume, a la imaginación una actividad propia y distintiva que la convierte en una facultad de concebir en la libertad que

[37] Relaciones que funcionan como principio unificador de las ideas. «Las cualidades de la que surge tal asociación y or las que es llevada la mente de este modo de una idea a otros son tres: semejanza, contiguidad en tiempo y lugar y causa y efecto». Hume, D. Tratado cit., p 99. [38] Hume, D. Investigación sobre el entendimiento humano. Recuperado el 29 de julio de 2014, de: http://www.sanfelipe.edu.uy/imgs/documentos/882_1.pdf. 2008 p. 9

[39] Ibídem, p. 10.

Thémata. Revista de Filosofía ํ53 (2016) pp.: 63-86. 
Einstein poseía, aunque tenga ésta por limite lo lógicamente posible y que por tanto, aquello que imaginemos, aunque "nunca haya sido visto u oído", incluya ya la idea de su posible existencia. Los monstruos pueden surgir según el autor inglés entonces como:

...una colección de ideas simples unidas por la imaginación y que poseen un nombre particular asignado a ellas (...), pero la diferencia entre estas ideas consiste en que las cualidades particulares que forman una sustancia son referidas por lo común a un algo desconocido en que se supone inhieren. ${ }^{40}$

Así, una idea como la de substancia, como nos ejemplifica Hume en el libro I- pero no menos la del tiempo-, deducimos a partir de la lectura global del Treatise, es "algo desconocido" al que referimos las ideas simples, y que aunque esta fuera absurda o no tuviera lugar, todavía podríamos salvar en su noción suponiendo la existencia de estas relaciones conectivas con las que opera ${ }^{41}$. Es por ello que la imaginación, más que formar conceptos o ideas, creemos que las supone ${ }^{42}$, y solo a partir de este facultad de actuar libremente pueden éstas devenir ficciones.

Suponer el tiempo, por ello, no puede ir más allá de imaginar un concepto que ha de contener la sucesión de ideas e impresiones percibidas desde nuestra situación particular, creando, esos sí a partir de aquí, un juego de conexiones extensivo que nos permita utilizarlo. Por eso Einstein mirando finalmente desde la lejanía de su autobiografía a su juventud puede decir que:

Cuando al recibir impresiones sensoriales, emergen imágenes de la memoria, no se trata aun de pensamiento. Cuando esas imágenes formen secuencias, cada una de las cuales evoca a otra, sigue sin poderse hablar de pensamiento. Pero cuando una determinada imagen reaparece en muchas de esas secuencias, se torna, precisamente en virtud de su recurrencia, un elemento ordenador de tales sucesiones, conectando secuencias que de suyo eran inconexas. Un elemento semejante se convierte en herramienta, en concepto. ${ }^{43}$

[40] Hume, D. Tratado sobre la naturaleza humana. Editora Nacional. Madrid, 1981 p. 105.

[41] Las mencionadas anteriormente operaciones de semejanza, contigüidad y causalidad.

[42] Es interesante la influencia de Berkeley en este concepto de imaginación: «Con relación a las ideas abstractas o generales se ha suscitado un problema muy importante: si son generales o particulares en la concepción que la mente se hace de ellas. Un gran filosofo (Berkeley) ha combatido la opinión tradicional sobre este asunto, afirmando que todas las ideas generales no son sino ideas particulares añadidas a un cierto término que les confiere mayor extensión, y que hace que recuerden ocasionalmente a otros individuos similares a ellas». Ibídem, p. 106.

[43] Einstein, A. Autobiografía y escritos científicos. Barcelona: Biblioteca Universal del Cír-

Thémata. Revista de Filosofía №53 (2016) pp.: 63-86. 
Pensar es un juego entonces que practicamos libremente con los conceptos, y cuya justificación, «reside en el grado de comprensión que con su ayuda podemos adquirir sobre las experiencias de los sentidos» ${ }^{44}$. Mientras que la verdad que estos pueden representar, nos dice el ahora científico convertido en pensador contemporáneo, queda lejos de este juego, a menos que exista un general consenso acerca de los elementos y las reglas del mismo ${ }^{45}$. Sin duda, Einstein nos ha propuesto las suyas en forma de unos postulados que, imaginados como destellos de luz vistos desde los andenes, nos han permitido deducir esta nueva herramienta que es su concepto de tiempo.

\section{Bibliografía}

Einstein, A. Autobiografía y escritos científicos. Madrid: Círculo de Lectores. Biblioteca Universal, 1995.

Einstein, A. Física i realitat (i altres escrits filosòfics). Santa coloma de Gramanet: Obrador Edéndum S.L, 2005

Einstein, A. La teoría de la relativitat $i$ altres textos. Institut d'Estudis Catalnas/Editorial Pórtic/ Eumo Editorial, 1998.

Einstein, A. Sobre la teoría de la relatividad y otras aportaciones. José M. Álvarez y Ana Goldar (Trads). Madrid: Editoral Sarpe, 2008.

Gamow, G. Biografía de la física. Estella: Salvat, 1971.

Hume, D. Tratado sobre la naturaleza humana. Madrid: Editora Nacional., 1981.

Mach, E. Análisis de las sensaciones. Barcelona: Alta Fulla, 1987.

Mach, E. Desarrollo histórico crítico de la mecánica. Buenos Aires: Espasa Calpe, 1949.

Merleau-Ponty, J. Einstein. París: Flammarion, 1993. Traducción cast. Sanz Sáenz, A. Albert Einstein: vida, obra y filosofia. Madrid: Alianza, 1994.

Mansur, J. C. Einstein, crítico del empirismo. Estudios 107, vol. xi, invierno 2013.

Newton, I. Principios matemáticos de la filosofia natura. Madrid: Editora Nacional, 1982.

culo de Lectores, 1995 p. 43

[44] Ibídem, p. 43.

[45] Ídem, p. 44.

Thémata. Revista de Filosofía №53 (2016) pp.: 63-86. 
Norton, John D. «How Hume and Mach Helped Einstein Find Special Relativity". In: Discourse on a New Method: Reinvigorating the Marriage of History and Philosophy of Science. Open Court, Chicago and La Salle, IL, 2010 pp. 359-386.

Robert Schulmann, A. J. Kox, Michel Janssen y József Illy, eds. The Collected Papers of Albert Einstein, vol. 8 (The Berlin Years: Correspondence,1914-1917), Parte A . Princeton: Princeton University Press, 1998.

Holton, G. "On the origins of the special theory of relativity». In: American Journal of Physics, 28, 1960, pp. 627-636, 633-636.

Sánchez Ron, JM (1983) .El origen y desarrollo de la relatividad. Madrid: Alianza.

Sánchez Ron, J. M. Einstein y la filosofía del siglo XX. Arbor Ciencia, Pensamiento y Cultura. CLXXXIII 728 noviembre-diciembre 2007 pp. 833-853, 2007.

Tipler, P.A. y Mosca, G (2010) Física Moderna. Barcelona: Editorial Reverte, 2010.

Wilbanks, J. Hume's theory of Imagination. Editorial: Pringer Verlag GMBH 01/07/2014. 
